Arq. Bras. Med. Vet. Zootec., v.67, n.4, p.1087-1095, 2015

\title{
Análise produtiva e econômica de suínos criados nos sistemas wean-to-finish e convencional de produção
}

\author{
[Productive and economic analysis of pigs raised in wean-to-finish and \\ conventional production systems]
}

\author{
W. Consoni, J. Cristani, F. Klaumann, P.M. Arruda, A.T. Zimmermann, R.G. Lorenzetti, \\ T.M. Dacoregio, A. Thaler Neto, S.D. Traverso
}

Centro de Ciências Agroveterinárias - Universidade do Estado de Santa Catarina - UDESC - Lages, SC

\begin{abstract}
RESUMO
O modelo de produção de suínos denominado Wean-to-Finish (WF) é considerado uma alternativa que busca alcançar melhor desempenho produtivo, redução de estresse aos animais e melhorias na logística. Nesse sistema, os leitões são desmamados e transferidos diretamente a um galpão WF (crechecrescimento-terminação), onde permanecem até o abate. Com o objetivo de avaliar os comportamentos produtivo e econômico de suínos alojados no sistema WF em comparação ao sistema convencional de produção $(\mathrm{CC})$, foram avaliados dois grupos de suínos provenientes das mesmas unidades produtoras de leitões, nascidos e desmamados na mesma semana. Um grupo foi alojado em uma creche do sistema convencional de produção (grupo CC), e o outro em um galpão WF adaptado (grupo WF), ambos em baias coletivas. Para análise de produção, foram calculados o consumo médio diário de ração (CMDR), o ganho de peso médio diário (GPMD) e a conversão alimentar (CA). Para análise econômica, foi determinado o custo por quilograma de ganho de peso vivo. Os dados foram submetidos à análise de variância utilizando-se o pacote estatístico SAS, previamente testados para normalidade dos resíduos pelo teste de Shapiro-Wilk, e as médias dos grupos comparadas pelo teste de Tukey (5\%). Na fase de creche, o GPMD foi de 0,468 e 0,449, e a CA de 1,48 e 1,42 para os Grupos WF e CC, respectivamente. Nas fases de crescimento e terminação, o GPMD foi de $0,924 \mathrm{~kg}$ no grupo WF e de $0,909 \mathrm{~kg}$ no grupo CC. A conversão alimentar foi de 2,41 para ambos os grupos, não sendo diferentes estatisticamente entre si $(\mathrm{P}>0,05)$ nas diferentes fases de produção nos parâmetros analisados. No aspecto econômico, o grupo WF apresentou os menores custos, com diferença de R $\$ 0,08$ por quilograma de ganho de peso vivo em comparação ao grupo CC. Os resultados do desempenho produtivo associado ao aspecto econômico indicam que o sistema WF pode ser uma alternativa viável dentro da cadeia produtiva de suínos.
\end{abstract}

Palavras-chave: redução de estresse, logística, análise econômica

\begin{abstract}
The pig production model called "Wean-to-Finish" (WF) is considered an alternative to better productive performance, reducing stress to the animals and improvements in logistics. In this system, the piglets are weaned and transferred directly to a WF shed (nursery-grower-finisher), where they remain until slaughter. Aiming to evaluate the productive and economic behavior of pigs housed in the WF system compared to conventional production system $(C C)$, two groups of pigs from the same sow farm, born and weaned in the same week were evaluated. One group was housed in a conventional nursery production system (CC group) and the other in a WF shed adapted (WF group), both in collective pens. For analysis of the average production, average daily feed intake (ADFI), average daily weight gain (ADWG) and feed conversion ratio $(F C R)$ were calculated. For economic analysis the cost per kilogram of live weight gain was determined. Data were subjected to analysis of variance using the SAS statistical package, previously tested for normality of the residuals using the Shapiro-Wilk test, and the means of groups were compared by the Tukey test $(5 \%)$. In the nursery phase ADWG was 0.468 and 0.449 , and the FCR was 1.48 and
\end{abstract}

Recebido em 2 de outubro de 2014

Aceito em 27 de março de 2015

E-mail:consonivet@hotmail.com 
1.42 for the WF and CC groups respectively. During the growing and finishing ADWG was $0.924 \mathrm{~kg}$ in the WF group and 0.909 in the CC group. The FCR was 2.41 for both groups, not being statistically different from each other $(P>0.05)$ at different stages of production in the analyzed parameters. In the economic aspect, the WF group had the lowest costs, with a difference of $R \$ 0.08$ per kilogram of live weight gain compared to the CC group. The results of productive performance associated with the economic aspect indicate that the WF system can be a viable alternative in the pig production chain.

Keywords: reducing stress, logistic, economic analysis

\section{INTRODUÇÃO}

O modelo de produção de suínos denominado Wean-to-Finish (WF) surgiu como uma alternativa para integradoras e produtores rurais, a fim de se alcançar melhor desempenho produtivo e sanitário, melhorias na logística, redução de estresse nos animais, consequentemente melhor resultado econômico para ambos. Os leitões são desmamados e transferidos diretamente a um galpão WF, onde permanecem até o abate (Dhuyvetter et al., 2012).

Estudos que compararam o WF aos sistemas convencionais de criação nos Estados Unidos (Connor, 1998) e no Chile (Peralta, 2008) relataram vantagens no desempenho dos animais a favor do sistema WF, com melhor ganho de peso médio diário (GPMD) e melhor conversão alimentar (CA), contudo Brumm et al. (2002), nos EUA, não observaram diferença de desempenho entre os dois sistemas em relação ao GPMD, à CA, ao consumo médio de ração (CMDR) e à qualidade de carcaça.

No Brasil, esse sistema começou a ser utilizado em 2008, e em 2009, foi realizado um acompanhamento sanitário de 3545 suínos, o qual constatou que a incidência e o período de ocorrência das doenças em granjas onde se utiliza o sistema de WF são semelhantes aos relatados em animais mantidos em sistemas convencionais (Gabardo et al., 2013), porém não existem dados produtivos e econômicos comparando os dois sistemas de produção. Este trabalho realizou análises de desempenho produtivo e econômico, comparando o sistema WF com o sistema convencional de produção. Os animais foram alojados no verão, e foi avaliada a viabilidade da implantação do sistema como uma alternativa no cenário nacional para a cadeia produtiva de suínos.

\section{MATERIAL E MÉTODOS}

Este trabalho foi aprovado pelo Comitê de Ética em Experimentação Animal (CETEA) do CAVUDESC de Lages - SC, protocolo número 1.24.12. Foram avaliados dois grupos de suínos, provenientes de três unidades de produção de leitões (UPLs), da região sul do estado de Santa Catarina, nascidos e desmamados na mesma semana, com idade média de 28 dias. O desmame dos animais ocorreu no início de março, compreendendo época de temperaturas quentes durante a fase de creche. Os animais foram identificados, pesados e alojados na fase de creche. Foram selecionados aproximadamente 400 animais de cada uma das três UPLs, dividindo-os em metade para cada grupo. Um dos grupos foi alojado em um galpão WF adaptado (grupo WF, com 600 animais) e o outro em um galpão de creche do sistema convencional de criação (grupo CC com 588 animais).

No alojamento, os leitões foram pesados individualmente e separados nas baias de acordo com sexo e faixa inicial de peso, seguindo o critério: $\leq 7,0 \mathrm{~kg}$, de 7,1 a $8,0 \mathrm{~kg}$ e $\geq 8,1 \mathrm{~kg}$. No grupo WF, os animais ficaram alojados em 20 baias, com 30 leitões cada, e no crechário convencional foram separados em 14 baias de 42 animais. Cada baia foi considerada uma unidade experimental, e os animais foram manejados conforme a rotina das granjas.

As fases de crescimento e terminação englobaram o período de outono e de início de inverno, com temperaturas mais baixas. Para a fase de crescimento, 390 animais do grupo CC foram transferidos para um galpão de crescimento e terminação do sistema convencional. No galpão WF adaptado, permaneceram 410 animais para a avaliação, conforme a capacidade da granja.

Para a fase de terminação, nos dois sistemas, os animais foram agrupados em baias inicialmente 
com 16 leitões. Após dois intervalos regulares de 15 dias, formaram-se baias de 14 animais no primeiro intervalo e de 12 após o segundo, com a realocação dos animais retirados para baias vazias, onde permaneceram sem movimentação até atingirem peso de abate. As rações foram formuladas para atender as exigências nutricionais dos animais segundo Rostagno et al. (2005), e os dois grupos receberam as mesmas dietas.

Para análise de desempenho produtivo, foram realizadas as pesagens individuais dos animais em cada troca de ração, que correspondeu aos períodos analisados durante todo o experimento. Ao todo, foram realizadas quatro pesagens na fase de creche e quatro pesagens nas fases de crescimento e terminação. Os dias das pesagens e a idade dos animais encontram-se na Tab. 1.

Tabela 1. Dias de alojamento, idade e número da pesagem dos animais durante a fase experimental

\begin{tabular}{|c|c|c|}
\hline $\begin{array}{c}\text { Dia de } \\
\text { alojamento }\end{array}$ & $\begin{array}{c}\text { Idade dos } \\
\text { animais (dias) }\end{array}$ & $\begin{array}{c}\mathrm{N}^{\circ} \mathrm{da} \\
\text { pesagem }\end{array}$ \\
\hline $1^{\circ}$ & 28 & $1^{\circ}$ \\
\hline $12^{\circ}$ & 39 & $2^{o}$ \\
\hline $27^{\circ}$ & 54 & $3^{\circ}$ \\
\hline $36^{\circ}$ & 63 & $4^{\circ}$ \\
\hline $63^{\circ}$ & 90 & $5^{\circ}$ \\
\hline $99^{\circ}$ & 126 & $6^{\circ}$ \\
\hline $126^{\circ}$ & 153 & $7^{\circ}$ \\
\hline $141^{\circ}$ & 168 & $8^{\circ}$ \\
\hline
\end{tabular}

Para o controle do consumo de ração durante a fase de creche, as rações eram pesadas antes do fornecimento, e as sobras eram pesadas diariamente e a cada troca de ração.

Nos períodos de crescimento e terminação, as rações foram fornecidas com restrição, quatro vezes ao dia, sendo a quantidade fornecida de acordo com a tabela de arraçoamento proposta pelo sistema de integração. Para controlar a quantidade fornecida nesses períodos, foram acopladas balanças digitais nos carrinhos de arraçoamento. Dessa forma, a ração foi pesada a cada fornecimento, em cada baia, de acordo com o número total de animais da baia. Com esses dados, foi possível obter o consumo médio diário de ração (CMDR) em kg, o ganho de peso médio diário (GPMD) em kg e a conversão alimentar
(CA), calculada pela razão entre CMDR e GPMD.

A análise da viabilidade econômica foi realizada com base no custo por quilograma de suíno produzido. Inicialmente foi calculado o custo da ração por quilograma de ganho de peso vivo. Os preços dos ingredientes utilizados na elaboração dos custos das rações foram coletados na região oeste de Santa Catarina, no período de realização do experimento. Também foram mensurados os gastos variáveis como mão de obra, limpeza e desinfecção, transportes e medicamentos.

Para análise estatística, foi utilizado o delineamento inteiramente ao acaso. Os dados foram submetidos à análise de variância, utilizando-se o modelo linear generalizado (GLM) do pacote estatístico SAS (SAS 9.1. SAS Institute, Cary, NC, USA), foram previamente testados para normalidade dos resíduos pelo teste de Shapiro-Wilk (Shapiro e Wilk, 1965), e compararam-se as médias dos grupos pelo teste de Tukey $(5 \%)$.

Os dados de desempenho produtivo foram analisados em sete diferentes intervalos de idade, nos períodos de creche e de crescimento e terminação, conforme a Tab. 2.

Tabela 2. Períodos de avaliação do desempenho produtivo nas diferentes idades, na fase de creche e nas fases de crescimento e terminação

\begin{tabular}{cc}
\hline Períodos & Idade em dias \\
\hline 1 & 28 a 39 \\
2 & 40 a 54 \\
3 & 55 a 63 \\
Creche total & 28 a 63 \\
4 & 64 a 90 \\
5 & 91 a 126 \\
6 & 127 a 153 \\
7 & 154 a 168 \\
Crescimento/terminação total & 64 a 168 \\
\hline
\end{tabular}

Para análise econômica, os dados obtidos dos custos de produção foram analisados estatisticamente para cada grupo avaliado com base no custo por quilograma de suíno produzido. Tanto para a análise produtiva quanto para a econômica, a baia foi considerada como uma unidade experimental. 


\section{Consoni et al.}

\section{RESULTADOS}

O galpão do sistema WF (Fig. 1) possuía divisórias de concreto entre as baias, piso compacto com declividade de $2,5 \%$ e lâmina d'água. Cada baia possuía dois bebedouros do tipo chupeta com regulagem de altura e comedouros linear de concreto com divisórias metálicas.

No galpão de creche do sistema convencional (Fig. 2), as baias eram suspensas com piso de plástico vazado e separadas por grades metálicas, possuíam quatro bebedouros tipo chupeta com regulador de altura e comedouros de madeira com divisórias individuais para os leitões. Ambos os galpões possuíam cortinado duplo e um forno à lenha para auxiliar no aquecimento e na manutenção da temperatura durante a fase de creche. O galpão das fases de crescimento e terminação do sistema convencional (Fig. 3) era semelhante ao galpão WF, com cortinado duplo, diferindo apenas por ser uma construção mais nova, de corredor com piso mais elevado.

$\mathrm{Na}$ pesagem inicial dos animais na fase de creche, no alojamento, as médias de peso foram semelhantes, sendo de $7,49 \mathrm{~kg}$ e de 7,38 para os grupos WF e CC, respectivamente. Os dados das pesagens dos animais, por grupo, durante a fase de creche, constam na Tab. 3 .

O grupo WF apresentou maior média de peso vivo em relação ao grupo CC aos 54 e 63 dias de idade $(\mathrm{P}<0,05)$.

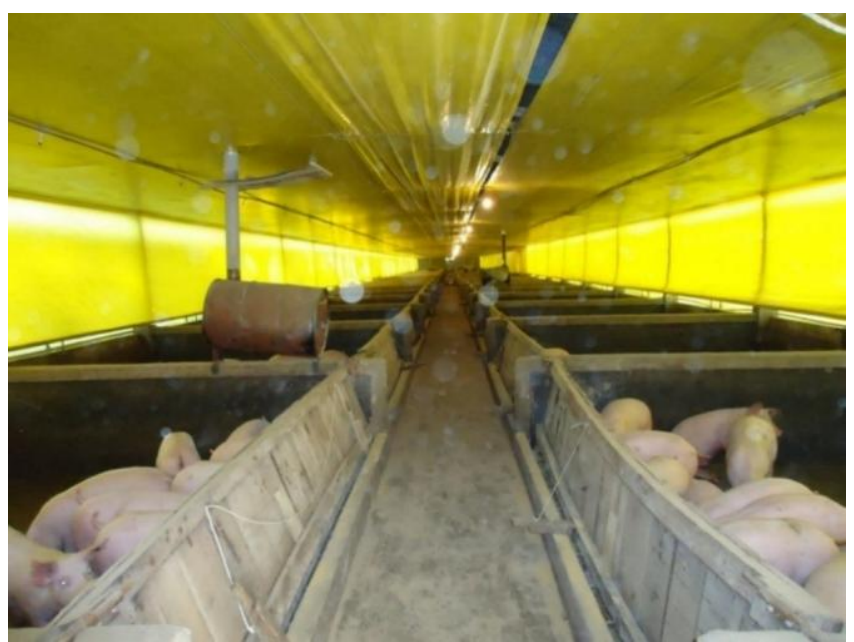

Figura 1. Galpão WF: cortinado duplo e forno à lenha para aquecimento durante o período de creche.

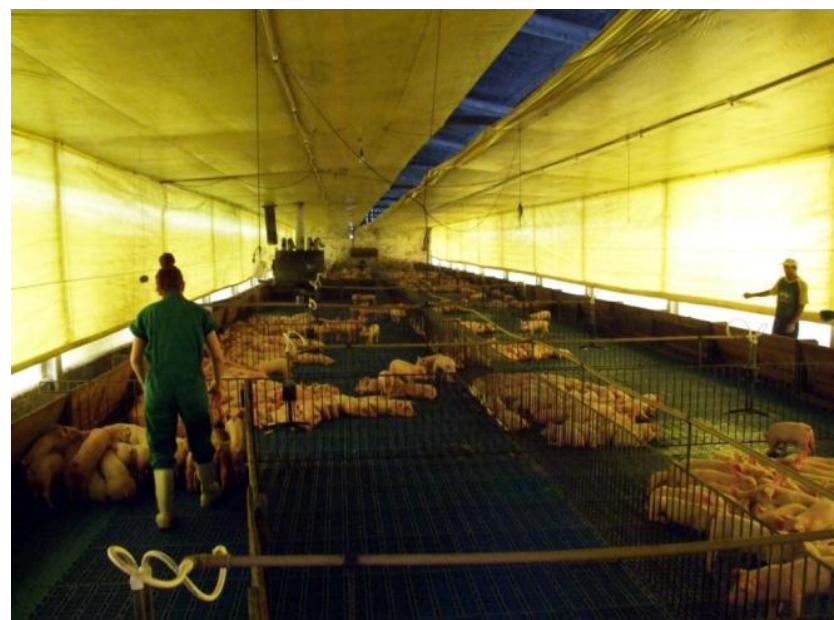

Figura 2. Galpão de creche do sistema convencional com piso elevado, cortinado duplo e forno para aquecimento dos animais. 


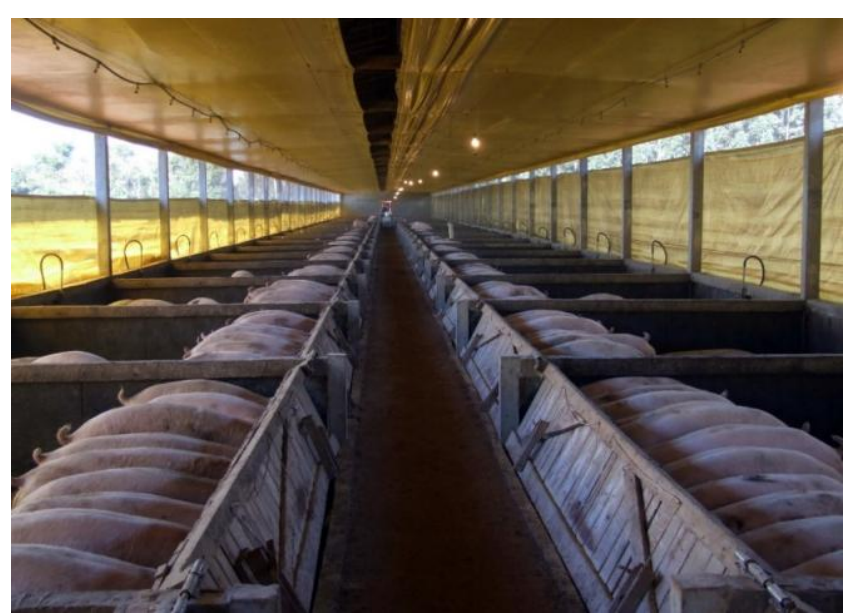

Figura 3. Galpão de crescimento e terminação do grupo CC.

Tabela 3. Peso médio dos animais por grupo na fase de creche em kg

\begin{tabular}{ccccc}
\hline Grupo & Peso 1 (28d) & Peso 2 (39d) & Peso 3 (54d) & Peso 4 (63d) \\
\hline WF & 7,49 & 10,19 & $17,80 \mathrm{a}$ & $24,14 \mathrm{a}$ \\
CC & 7,38 & 10,50 & $17,15 \mathrm{~b}$ & $23,55 \mathrm{~b}$ \\
$P$ & 0,5622 & 0,6076 & 0,0371 & 0,0174 \\
\hline
\end{tabular}

Letras diferentes nas colunas representam diferença significativa $(\mathrm{P}<0,05)$.

O CMDR, o GPMD e a CA, na fase de creche para os diferentes grupos, podem ser observados na Tab. 4.

Houve diferença significativa tanto para o CMDR quanto para o GPMD $(\mathrm{P}<0,05)$, com exceção do CMDR1. Os valores foram maiores para o grupo WF a partir do segundo período. Nos CMDR e GPMD totais da fase de creche, também houve diferença significativa, com vantagem para o grupo WF.

Tabela 4. Consumo médio diário de ração (CMDR), ganho de peso médio diário (GPMD) e conversão alimentar (CA) em kg, nos períodos 1 (28 a 39), 2 (40 a 54), 3 (55 a 63) e total (28 a 63), na fase de creche

\begin{tabular}{ccccc}
\hline Grupo & Período & WF & CC & $P$ \\
\hline \multirow{2}{*}{ CMDR } & 1 & 0,308 & 0,344 & 0,0003 \\
& 2 & $0,704 \mathrm{a}$ & $0,645 \mathrm{~b}$ & 0,0003 \\
& 3 & $1,093 \mathrm{a}$ & $0,847 \mathrm{~b}$ & $<0,0001$ \\
& $28 \mathrm{a} 63$ dias & $0,701 \mathrm{a}$ & $0,612 \mathrm{~b}$ & 0,0425 \\
\hline \multirow{2}{*}{ GPMD } & 1 & $0,247 \mathrm{~b}$ & $0,295 \mathrm{a}$ & 0,0006 \\
& 2 & $0,511 \mathrm{a}$ & $0,449 \mathrm{~b}$ & $<0,0001$ \\
& 3 & $0,632 \mathrm{a}$ & $0,547 \mathrm{~b}$ & $<0,0001$ \\
& $28 \mathrm{a} 63$ dias & $0,468 \mathrm{a}$ & $0,449 \mathrm{~b}$ & 0,0024 \\
\hline \multirow{2}{*}{ CA } & 1 & $1,26 \mathrm{a}$ & $1,16 \mathrm{~b}$ & 0,0266 \\
& 2 & 1,38 & 1,43 & 0,0587 \\
& 3 & $1,73 \mathrm{a}$ & $1,56 \mathrm{~b}$ & 0,0048 \\
& $28 \mathrm{a} 63$ dias & $1,48 \mathrm{a}$ & $1,42 \mathrm{~b}$ & 0,0068 \\
\hline
\end{tabular}

Letras diferentes nas linhas representam diferença significativa $(\mathrm{P}<0,05)$.

Em relação à conversão alimentar, os grupos diferiram significativamente nos períodos 1 e 3 , sendo estes os períodos de melhor CA do grupo $\mathrm{CC}$ em relação ao grupo $\mathrm{WF}$, assim como a CA total. No período 2, não houve diferença estatística entre os grupos para a CA.
Para as fases de crescimento e terminação, o peso médio dos animais encontra-se na Tab. 5. Os dados de CMDR, GPMD e CA podem ser observados na Tab. 6. 
Tabela 5. Peso médio individual dos animais em kg nos grupos wean-to-finish (WF) e convencional de criação (CC) nas idades de 63, 90, 126, 154 e 168 dias

\begin{tabular}{cccccc}
\hline Grupo & Peso 63d & Peso 90d & Peso 126d & Peso 154d & Peso 168d \\
\hline WF & 24,36 & 45,18 & $77,89 \mathrm{a}$ & $102,32 \mathrm{a}$ & $119,93 \mathrm{a}$ \\
CC & 23,66 & 44,10 & $73,95 \mathrm{~b}$ & $99,84 \mathrm{~b}$ & $116,13 \mathrm{~b}$ \\
$P$ & 0,0865 & 0,1205 & $<0,0001$ & 0,0063 & 0,0003 \\
\hline
\end{tabular}

Letras diferentes nas colunas representam diferença significativa $(\mathrm{P}<0,05)$.

Houve diferença significativa em relação ao peso médio a partir dos 126 dias de idade e nas pesagens seguintes (154 e 168 dias de idade), com vantagem para os animais do grupo do WF.
Para o CMDR, houve diferença significativa para os períodos 4, 5 e 7 , que se apresentaram superiores no grupo WF, assim como para o valor total das fases de crescimento e terminação.

Tabela 6. Consumo médio diário de ração (CMDR), ganho de peso médio diário (GPMD) e conversão alimentar (CA) em kg, nos períodos 4 (64 a 90), 5 (91 a 126), 6 (127 a 153), 7 (154 a 168) e total (64 a 168), nas fases de crescimento e terminação.

\begin{tabular}{lcccc}
\hline Grupo & Período & WF & CC & P \\
\hline \multirow{3}{*}{ CMDR } & 4 & $1,359 \mathrm{a}$ & $1,352 \mathrm{~b}$ & $<0,0001$ \\
& 5 & $1,908 \mathrm{a}$ & $1,834 \mathrm{~b}$ & $<0,0001$ \\
& 6 & 2,433 & 2,447 & 0,3544 \\
& 7 & $3,218 \mathrm{a}$ & $3,125 \mathrm{~b}$ & $<0,0001$ \\
GPMD & 64 aos 168 & $2,229 \mathrm{a}$ & $2,190 \mathrm{~b}$ & 0,0399 \\
\hline & 4 & 0,777 & 0,776 & 0,8968 \\
& 5 & $0,905 \mathrm{a}$ & $0,834 \mathrm{~b}$ & $<0,0001$ \\
& 6 & 0,955 & 0,957 & 0,9216 \\
& 7 & 1,062 & 1,083 & 0,6823 \\
& 64 aos 168 & $0,924 \mathrm{a}$ & $0,909 \mathrm{~b}$ & 0,0140 \\
\hline \multirow{3}{*}{ CA } & 4 & 1,75 & 1,74 & 0,4699 \\
& 5 & $2,11 \mathrm{~b}$ & $2,20 \mathrm{a}$ & 0,0001 \\
& 6 & 2,57 & 2,57 & 0,9999 \\
& 7 & 3,04 & 2,95 & 0,4584 \\
& 64 aos 168 & 2,41 & 2,41 & 0,6207 \\
\hline
\end{tabular}

Letras diferentes nas linhas representam diferença significativa $(\mathrm{P}<0,05)$.

Em relação ao GPMD e à $\mathrm{CA}$, os grupos diferiram entre si no período 5, com vantagem para os animais dos grupos WF. O grupo WF apresentou melhor GPMD total das fases de crescimento e terminação em relação ao grupo CC. Em relação à CA total, os grupos não diferiram entre si.

No aspecto econômico, o grupo CC apresentou os custos para transportes de animais, mão de obra, limpeza e desinfecção na transferência entre as fases de creche para crescimento/terminação. Os gastos com transportes foram de $\mathrm{R} \$ 40,00$ por tonelada de suíno transportada, e o custo médio por granja do programa de limpeza e desinfecção e mão de obra foi de $\mathrm{R} \$ 438,00$.
Os custos com medicação na fase de creche foram de $\mathrm{R} \$ 0,828$ e $\mathrm{R} \$ 1,319$ por animal para os grupos WF e CC, respectivamente. Nas fases de crescimento e terminação, foram de $\mathrm{R} \$ 4,37$ por animal para o grupo $\mathrm{CC}$ e de $\mathrm{R} \$ 5,54$ por animal para o grupo WF. A diferença final entre os grupos na fase de creche foi de aproximadamente $\mathrm{R} \$ 0,06$, e o gasto com medicamentos no crechário do sistema convencional durante esse período foi de $\mathrm{R} \$ 0,037$ a mais por quilograma produzido em relação ao grupo WF.

Os custos totais por quilograma de suíno produzido e o percentual das variáveis nos custos de produção podem ser observados nas Tab. 7 e 8. 
Análise produtiva...

Tabela 7. Custos totais por quilograma de suíno produzido nos diferentes grupos

\begin{tabular}{cccc} 
Grupo & Creche & Cresc./Term. & Total \\
\hline $\mathrm{WF}$ & $\mathrm{R} \$ 1,76 \mathrm{~b}$ & $\mathrm{R} \$ 1,74 \mathrm{~b}$ & $\mathrm{R} \$ 3,50$ \\
$\mathrm{CC}$ & $\mathrm{R} \$ 1,82 \mathrm{a}$ & $\mathrm{R} \$ 1,76 \mathrm{a}$ & $\mathrm{R} \$ 3,58$ \\
$P$ & 0,0226 & 0,0276 & - \\
\hline
\end{tabular}

Letras diferentes nas colunas representam diferença significativa $(\mathrm{P}<0,05)$.

Os resultados econômicos diferiram entre si o grupo WF apresentou menores valores de $(\mathrm{P}<0,05)$ tanto na fase de creche quanto nas fases de crescimento e terminação. Em ambas as fases, custos para produção de quilogramas de suíno.

Tabela 8. Percentual das variáveis no custo total por quilograma de suíno produzido (\%)

\begin{tabular}{lcccc} 
& \multicolumn{2}{c}{ Creche } & \multicolumn{2}{c}{ Cresc./term. } \\
\cline { 2 - 5 } \multicolumn{1}{c}{ Variável } & WF & CC & WF & CC \\
\hline Ração & 94.5 & 92.4 & 96.7 & 96.3 \\
PLD e mão de obra & 2.6 & 2.7 & 0.0 & 0.4 \\
Medicamentos & 2.9 & 4,9 & 3.3 & 2.7 \\
Transportes &. &. & 0.0 & 0.6 \\
\hline
\end{tabular}

\section{DISCUSSÃO}

Durante a fase de creche, a instalação utilizada no grupo WF não seguia o padrão recomendado pela literatura, com adaptação de piso e fornecimento de calor direto para os leitões (Hollis, 2007). O piso utilizado era compacto e o fornecimento de calor baseava-se apenas nos fornos, o que não foi suficiente para manter a temperatura interna ideal para a fase, a qual apresentou oscilação térmica de $8^{\circ} \mathrm{C}$, submetendo os animais a estresse térmico. Esses fatores podem ter influenciado no desempenho inicial dos leitões, especialmente na primeira semana de alojamento dentro do galpão WF. Dentro da zona de conforto térmico, a energia da dieta é utilizada para crescimento, manutenção e atividade física; abaixo dessa temperatura, energia adicional é necessária para manter a homeotermia (Collin et al., 2001). Dessa forma, ocorre uma queda na produção, pois os animais deixam de utilizar energia para a produção, gastando-a para gerar calor.

A melhor conversão alimentar do grupo CC na fase de creche pode ser explicada principalmente pelo manejo realizado pelo produtor, que, no período final da fase, restringia a alimentação dos animais, pois a remuneração era baseada na conversão alimentar. Esse fato pode ser observado na tabela de consumo de ração, onde os valores do grupo CC são menores nos dois últimos períodos da fase de creche.

Os dados de GPMD e CA foram analisados e pôde-se concluir que, apesar da pior conversão alimentar apresentada pelo grupo WF, o aumento do fornecimento de ração na fase final, acompanhado de um aumento no GPMD e de maior peso médio final, compensou economicamente, sendo mais vantajosa a remuneração baseada especialmente no GPMD.

Nas fases de crescimento e terminação, o fornecimento de ração era realizado da mesma forma nas duas granjas, seguindo a tabela de arraçoamento sugerida pela integradora. Porém, houve diferença significativa para a quantidade de consumo de ração entre os grupos, com os animais do WF apresentando os maiores valores de CMDR. Esse maior consumo de ração resultou em melhores GPMD e peso médio final deste grupo. Também pode ser levado em consideração que os animais do grupo WF iniciaram a fase de crescimento com peso um pouco superior ao do grupo $\mathrm{CC}$, apesar de não apresentarem diferença estatística. Animais com peso mais leve ao alojamento tendem a apresentar menor peso ao abate quando comparados a animais mais pesados, pois não conseguem compensar a diferença inicial existente entre eles (Almeida et al., 2009). Além disso, os animais do grupo CC passaram pelo 
estresse do transporte, o que leva a uma diminuição no consumo de ração durante os primeiros dias de alojamento. No início das fases de crescimento e terminação, observou-se a ocorrência de diarreia em algumas baias do grupo CC. A ocorrência de doenças entéricas afeta especialmente a conversão alimentar, levando a um baixo crescimento e a um aumento de custos de produção (Chase-Topping et al., 2007).

Em relação ao aspecto econômico, ao final das duas fases houve vantagem significativa para o grupo WF em relação ao CC, com uma diferença de $\mathrm{R} \$ 0,08$ por quilograma de suíno produzido. Ao calcular-se o ganho médio dos animais do grupo WF durante todo o período de avaliação (creche/crescimento/terminação), que foi de aproximadamente $112 \mathrm{~kg}$ por animal, obtém-se um valor médio de $\mathrm{R} \$ 8.96$ por animal de redução de custos dentro do grupo WF. Nesse aspecto, o WF demonstrou-se vantajoso comparado ao sistema convencional.

Houve maior gasto com medicamentos na fase de creche no grupo $\mathrm{CC}$ devido à maior ocorrência de enfermidades no crechário convencional. Caso não ocorresse esse maior gasto, a diferença final seria de $\mathrm{R} \$ 0,053$ por quilograma produzido, ou seja, ainda assim, o WF se tornaria vantajoso economicamente em relação ao sistema convencional, com uma diferença final de $\mathrm{R} \$ 5,93$ por animal de redução de custos. Também pode ser analisado o maior gasto com medicamentos no grupo WF na fase final de crescimento e na de terminação, devido à ocorrência de diarreias, o que elevou o custo em aproximadamente $\mathrm{R} \$ 0,01$ por quilograma produzido, correspondendo a $\mathrm{R} \$ 1,17$ por animal de custo a mais para o grupo, porém esse fato não influenciou no resultado final, que, da mesma forma, aponta o WF como o sistema mais vantajoso economicamente.

A diferença final de custos em favor do sistema WF existe devido à não utilização de transportes na troca da fase da creche para as fases de crescimento e terminação. Além disso, a não transferência dos animais reduz o custo com limpeza e desinfecção e os dias de vazio sanitário, com otimização do tempo de ocupação da granja. Esses fatores no sistema WF representaram economia de aproximadamente $\mathrm{R} \$$ 0,019 por quilograma de suíno produzido, o que equivale a R\$ 2,12 por animal, levando em consideração o ganho do desmame até o abate, somatória que pode influenciar na adoção ou não desse sistema por uma integradora. A eliminação da saída dos animais do galpão de creche para o de crescimento/terminação, resultando em economia de transportes, limpeza e desinfecção, também foi relatada por Kummer et al. (2009) e Peralta (2008).

A subutilização do espaço no início do alojamento, ou seja, o espaço ocioso dentro das granjas que trabalham com o sistema WF, é mencionada como desvantagem desse sistema (Brumm et al., 2002; Peralta, 2008). Segundo Wolter et al. (2002), uma das formas de melhorar o aproveitamento e reduzir o custo fixo por área de galpão é a realização do duplo alojamento, como foi feito durante a execução do projeto. Para isso, é necessário que a integradora trabalhe com os dois sistemas de produção.

\section{CONCLUSÕES}

Durante a fase de creche, o grupo WF foi melhor em ganho de peso médio diário e pior em conversão alimentar em relação ao grupo $\mathrm{CC}$. Durante as fases de crescimento e terminação, o grupo WF foi melhor que o grupo $\mathrm{CC}$ em peso médio final e GPMD. Os dois grupos foram iguais em CA. O grupo WF foi mais vantajoso economicamente. Pode-se concluir, dessa forma, que o sistema wean-to-finish é uma alternativa viável dentro da cadeia produtiva de suínos.

\section{AGRADECIMENTOS}

Ao Conselho Nacional de Desenvolvimento Científico e Tecnológico (CNPq) e à Fundação de Amparo à Pesquisa e Inovação do Estado de Santa Catarina (FAPESC), pelo financiamento do projeto; à Secretaria do Estado da Educação (SED), pela concessão da bolsa de mestrado. 


\section{REFERÊNCIAS}

ALMEIDA, M.N.; GHELLER, N.B.; LIPPKE, R.T. et al. Fatores que contribuem para a falta de uniformidade de suínos. Acta Sci. Vet., v. 37, p. 31-37, 2009.

BRUMM, M. C.; BAYSINGER, A.K.; WILLS, R.W.; THALER, R.C. Effect of wean-to-finish management on pig performance. J. Anim. Sci., v. 80, p. 309-315, 2002.

CHASE TOPPING, M.E.; GUNN, G.; STRACHAN, W.D. et al. Epidemiology of porcine non-specific colitis on Scottish farms. Vet. J., v.173, p. 353-360, 2007.

COLLIN, A.; VAN MILGEN, J.; DUBOIS, S.; NOBLET, J. Effect of high temperature and feeding level on energy utilization in piglets. $J$. Anim. Sci., v.79, p.1849-1857, 2001.

CONNOR, J.F. Wean-to-finish construction alternatives management and performance. In: ALLEN D. LEMAN SWINE CONFERENCE, 35., 1998, Saint Paul. Proceedings... Saint Paul, University of Minessota, 1998.

DHUYVETTER, K.C.; TONSOR, G.T.; TOKACH, M. D. et al. Swine wean-to-finish: cost-return budget. Kansas: Kansas State University, 2012. 4p. (Farm Managenet Guide, 2757).

GABARDO, M.P.; ZANDONAI, A.D.; CAMARGO, M.C. et al. Caracterização sanitária de suínos criados em sistema "wean-to-finish". Med. Vet., v.7, p. 23-31, 2013.
HOLLIS, W.L. New Production Systems Weanto-Finish For Growning Pigs. In: CONGRESSO BRASILEIRO DE VETERINÁRIOS ESPECIALISTAS EM SUÍNOS, 13., 2007, Florianópolis. Anais... Concórdia: Embrapa, 2007. 819p.

KUMMER, R.; GONÇALVES, M.A.D.; LIPPKE, R.T. et al. Fatores que influenciam no desempenho dos leitões na fase de creche. Acta Sci. Vet., v.37, Supl 1, s195-s209, 2009.

PERALTA, W. Sistema destete venta en Chile. Acta Sci. Vet., v.36, Supl 1, p.131-136, 2008.

ROSTAGNO, H.S.; ALBINO, L.F.T.; DONZELE, J.L. et al. Tabelas brasileiras para aves e suínos: composição de alimentos $\mathrm{e}$ exigências nutricionais. Viçosa: UFV, 2005. 184 p.

SAS/STAT. ®. 9.1 User's. Guide. Cary, NC: SAS Institute, 2004.

SHAPIRO, S.S.; WILK, M.B. An analysis of variance test for normality (complete somplls). Brometrika, v.52, p.591-611, 1965.

WOLTER, B.F.; ELLIS, M.; DEDECKER, J.M. et al. Effects of double stocking and weighing frequency on pig performance in wean-to-finish production systems. J. Anim. Sci., v.80, p.14421450, 2002. 